T. E. Wessendorf', 2
G. Alymov ${ }^{1}$
Y.-M. Wang ${ }^{2}$
J. Stampa'
A. F. Thilmann ${ }^{2}$
H. Teschler ${ }^{1}$

\section{Pulsoximetrie als Schlafapnoescreening bei Schlaganfallpatienten}

\author{
Pulse Oximetry Screening for Sleep-disordered Breathing in Stroke
}

\section{Zusammenfassung}

Einleitung: Ein großer Teil aller Patienten mit Schlaganfall leidet an einer obstruktiven Schlafapnoe (OSA), die zum Zeitpunkt des akuten Ereignisses nicht bekannt ist. Zwar gilt die Polysomnographie (PSG) bei Verdacht auf OSA als diagnostischer Goldstandard, die routinemäßige Durchführung ist jedoch nicht praktikabel. Eine Pulsoximetrie könnte jedoch ein einfach anwendbarer und kostengünstiger Screeningtest sein. Methodik: Bei 184 Schlaganfallpatienten wurde das während der Polysomnographie (PSG) digital aufgezeichnetete pulsoximetrische Signal vollautomatisch mittels einer Software (AHDip) im Hinblick auf Desaturationen, definiert als Abnahme der Sauerstoffsättigung von $\geq 4 \%$, analysiert. Der Apnoe-Hypopnoe-Index (AHI) in der PSG wurde als Goldstandard definiert und mit der Anzahl der mit AHDip automatisch erkannten Sauerstoffentsättigungen (ODI) pro Stunde Aufzeichnungsdauer verglichen. Ergebnisse: Die Korrelation zwischen AHI in der PSG und Desaturationsindex (ODI) in der Pulsoximetrie betrug $r=0,84(p<0,001)$. In Abhängigkeit von der Definition einer relevanten schlafbezogenen Atmungsstörung (AHI 10, 15, 20 oder 30/h) und dem angewandten cut-offWert für den ODI (z.B. 15/h) ergaben sich für die Pulsoximetrie eine Sensitivität von 32-83\% und eine Spezifität von 99-96\%. Die diagnostische Genauigkeit (gemessen als area under the curve der ROC-Kurve) bei einem AHI $\geq 20$ als Diagnose einer SBAS betrug 96\%. Schlussfolgerung: Bei Patienten mit Schlaganfall ermöglicht die automatische Analyse des pulsoximetrischen Signals mittels AHDip eine realistische Erkennung schlafbezogener Atmungsstörungen und könnte allgemein in Populationen mit hoher Vortestwahrscheinlichkeit eingesetzt werden.

\section{Abstract}

Background: The prevalence of sleep-disordered breathing, particularly obstructive sleep apnea, among stroke patients is high. Routine screening with the current diagnostic gold standard of polysomnography is not feasible. Pulse oximetry could be a simple screening test. Methods: The signal of pulse oximetry, recorded during full polysomnography in 184 stroke patients during neurological rehabilitation, was analyzed automatically by software for desaturations $\geq 4 \%$. The polysomnographic apnoeahypopnoea-index (AHI) was used as the diagnostic gold standard and compared with the oxygen desaturation index (ODI). Results: Correlation between AHI of PSG and ODI of oximetry was $r=0.84(p<0.001)$. Dependent on the definition of SDB (AHI 10, 15,20 or 30/h) and the cut-off-point for the ODI (e.g. 15/h) sensitivity was $32-83 \%$ and specificity $99-96 \%$. The diagnostic accuracy as determined by the area under the ROC-curve was $96 \%$. Conclusion: Automated analysis of pulse oximetry gives reasonable results for screening for SDB in stroke patients and could be used in populations with high pre-test probability. 
Schlafbezogene Atmungsstörungen (SBAS) haben bei Patienten mit Schlaganfall eine hohe Prävalenz (25\% - 50\%) [1 -4]. Obwohl die klinische Relevanz derzeit noch nicht hinreichend geklärt ist, gibt es Hinweise auf eine erhöhte Morbidität und Mortalität dieser Patientengruppe [5,6]. Auch wenn kaum Tagessymptome vorliegen, profitiert ein Teil der Patienten von einer nasalen CPAP-Therapie [7,8]. In einer kürzlich erschienenen Übersichtsarbeit wird allein aufgrund der hohen Prävalenz eine routinemäßige Evaluation von Patienten mit Schlaganfall und TIA gefordert [9].

Die Polysomnographie als diagnostischer Goldstandard ist als Routineuntersuchung nach Schlaganfall aufwändig und nicht praktikabel. Ein einfaches Screening auf SBAS nach Schlaganfall wäre daher sinnvoll. Die Rolle der Pulsoximetrie als einfachstes apparatives Screening auf SBAS ist immer wieder diskutiert worden [10]. Vazquez et al. konnten zeigen, dass eine automatische Analyse des pulsoximetrischen Signals mit hinreichender Genauigkeit eine SBAS in einer Population mit hoher Vortestwahrscheinlichkeit diagnostizieren kann [11]. Wir haben daher die Validität eines pulsoximetrischen Screenings bei Schlaganfallpatienten in der neurologischen Rehabilitation untersucht.

\section{Methodik}

Für diese Untersuchung wurden die polysomnographischen Daten von 184 Patienten mit Schlaganfall analysiert, die im Schlaflabor der Fachklinik Rhein/Ruhr, Essen, untersucht wurden. Kein Patient erhielt eine Sauerstofftherapie. Unter diesen Patienten befanden sich 36 mit einem AHI $\geq 20 / h$ (19,6\%). Die Charakteristika der Patienten finden sich in Tab. 1.

Tab. 1 Patientencharakteristika

\begin{tabular}{|ll}
\hline Geschlecht $(\mathrm{m} / \mathrm{w})$ & $115 / 69$ \\
\hline Alter $($ Jahre $)$ & $62 \pm 9,4$ \\
\hline Body Mass Index $\left(\mathrm{kg} / \mathrm{m}^{2}\right)$ & $28 \pm 4,3$ \\
\hline AHI $(/ \mathrm{h})$ & $13 \pm 15,6$ \\
\hline Minimale $\mathrm{SaO}_{2}(\%)$ & $85 \pm 6,2$ \\
\hline Epworth-Skala $(\mathrm{n}=149)$ & $6 \pm 4,2$ \\
\hline
\end{tabular}

Die Polysomnographien (PSG) wurden nach üblichen Standards durchgeführt und sind an anderer Stelle im Detail beschrieben [3]. Der Atemstrom wurde mit einem Thermistor registriert. Als Kriterium für eine relevante Entsättigung galt eine Abnahme der Sauerstoffsättigung $\left(\mathrm{SaO}_{2}\right)$ von $\geq 4 \%$. Die Schlafstadien und Arousals wurden von einem Auswerter, der die Ergebnisse der Pulsoximetrie nicht kannte, manuell nach üblichen Kriterien ermittelt $[12,13]$.

Die im Rahmen der Polysomnographie mit einem Pulsoximeter Model 305A (ResMed, San Diego, USA) kontinuierlich gemessenen Werte für Sättigung und Puls wurden mit einer Datendichte von $1 \mathrm{~Hz}$ gespeichert. Nach Beendigung der Messung wurden die Daten über eine serielle Schnittstelle in einen handelsüblichen
Personalcomputer übertragen. Bei Verwendung eines NANOXPulsoximeters (Fenyves \& Gut, Hechingen) findet die Übertragung über die eingebaute Infrarotschnittstelle statt. Zur graphischen Darstellung der Messwerte im zeitlichen Verlauf und Erzeugung von Histogrammen und Tabellen zwecks numerischer Auflistung der Entsättigungen wurde eine Software (AHDip) entwickelt. Die Software gestattet in einem Menü für die Einstellungen die Änderung der Entsättigungsschwelle einzustellen, ab der ein Ereignis als relevant betrachtet wird. Voreingestellt und in dieser Studie verwendet war ein Sättigungsabfall von mindestens $4 \%$. Der Algorithmus bestimmt zunächst die Hüllkurve der höchsten Entsättigungswerte. Danach wird Messwert für Messwert geprüft, ob es zu einem Abfall der Sättigung gegenüber dem Hüllkurvenwert kommt und ob dabei der eingestellte Schwellenwert unterschritten wird. Falls dieses Ereignis kürzer als 90 Sekunden andauert, wird eine relevante Entsättigung registriert und zeitgerecht auf der X-Achse als Zeitmaßstab der Registrierung als Strich dargestellt, dessen Länge auf der Y-Achse in positiver Richtung der Dauer und in negativer Richtung dem Ausmaß der Entsättigung entspricht (Abb.1). Der Zeitfaktor für die maximale Dauer einer Entsättigung (in dieser Studie $90 \mathrm{Se}$ kunden) kann im Einstellmenü beliebig geändert werden. Änderungen der Sättigung binnen einer Sekunde werden als Artefakt gewertet und bei der automatischen Auswertung und graphischen Darstellung nicht berücksichtigt.

Für diese Analyse war das pulsoximetrische Rohsignal nicht verändert worden. Als Entsättigungsindex (ODI, oxygen desaturation index) wurde die Anzahl der Desaturationen pro Stunde Aufzeichnungszeit ermittelt. Der ODI wurde mit dem polysomnographisch ermittelten AHI verglichen.

\section{Statistik}

Die Korrelation zwischen AHI und ODI wurde mit dem Spearman-Koeffizienten ermittelt. Sensitivität, Spezifität, positiver und negativer Vorhersagewert sowie Likelihood ratios wurden in Abhängigkeit von der Definition einer SBAS (polysomnographisch ermittelter AHI von 10, 15, 20 oder 30/h) sowie bei verschiedenen cut-off-Werten für den ODI (5, 10, 15 oder 20/h) berechnet. Ein Bland-Altman-Plot [14] wurde zum Vergleich der beiden Methoden konstruiert. Die diagnostische Genauigkeit bei einem $A H I \geq 20$ als Definition einer SBAS wurde durch eine Receiver-Operating-Characteristic(ROC-)-Kurve ermittelt.

\section{Ergebnisse}

Der Korrelationskoeffizient zwischen AHI und Entsättigungsindex (ODI) der Pulsoximetrie beträgt $r=0,84$ ( $p<0,001$, Abb. 2).

Die Werte für Sensitivität, Spezifität, positiven und negativen Vorhersagewert (in \%) sowie die positive und negative Likelihood ratio sind in Tab. 2 in Abhängigkeit des Cut-off für den $\operatorname{ODI}(5,10$, 15 und 20/h) sowie für verschiedene polysomnographische Definitionen einer relevanten SBAS (AHI 10, 15, 20 oder 30/h) dargestellt.

Der Bland-Altman-Plot (Abb.3) ergibt eine Tendenz zur Unterschätzung des ODI bei hohen Werten für den AHI. Der Mittelwert 


\begin{tabular}{|c|c|c|}
\hline $\mathrm{SaO} 2$ & & \\
\hline$\%$ & $\%$ & $\min$ \\
\hline$<60$ & 0.0 & 0.0 \\
\hline$<65$ & 0.0 & 0.0 \\
\hline$<70$ & 0.0 & 0.0 \\
\hline$<75$ & 0.0 & 0.0 \\
\hline$<80$ & 0.2 & 0.8 \\
\hline$<85$ & 2.0 & 6.8 \\
\hline$<90$ & 19.4 & 66.3 \\
\hline$<95$ & 80.5 & 275.8 \\
\hline
\end{tabular}

Sättigungsverteilung

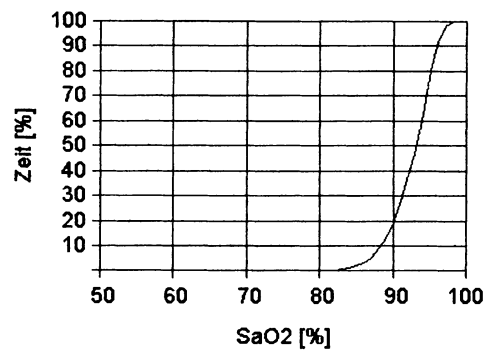

Entsättigungen

\begin{tabular}{r|r}
\hline Anzahl & 221 \\
Max. dSaO2 [\%] & 18 \\
Mittl. dSaO2 [\%] & 6.4 \\
Max. Dauer [s] & 60 \\
Mittl. Dauer [s] & 39.8
\end{tabular}

Pulsfrequenzverteilung

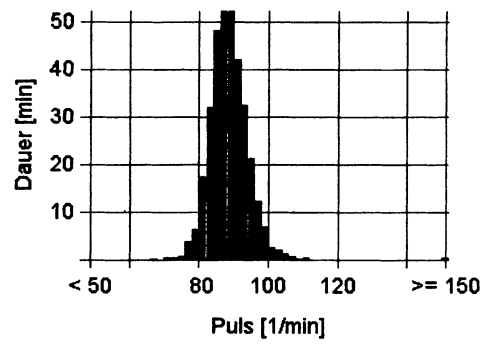

Abb. 1 Graphische Darstellung der mit AHDip analysierten Sättigungs- und Pulswerte eines Patienten mit obstruktiver Schlafapnoe: Die Entsättigungen sind auf der X-Achse als Zeitmaßstab der Registrierung als Strich dargestellt, dessen Länge auf der Y-Achse in positiver Richtung der Dauer (in s) und in negativer Richtung dem Ausmaß der Entsättigung (in \%) entspricht.

\section{Entsättigungen}

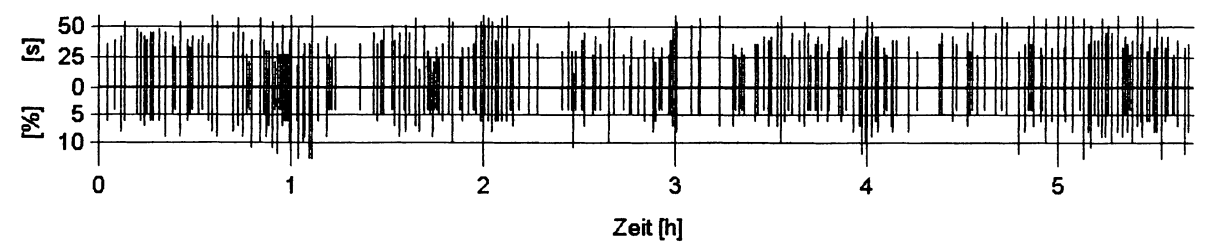

Tab. 2 Sensitivität, Spezifität, positiver (PPV) und negativer (NPV) Vorhersagewert (in \%) und Wahrscheinlichkeitsverhältnisse (Likelihood ratios) für ein positives (LR+) und ein negatives Testergebnis (LR-) in Abhängigkeit von AHI und ODI

\section{Spezifität}

\begin{tabular}{|c|c|c|c|c|c|c|c|}
\hline \multicolumn{8}{|l|}{ ODI } \\
\hline$\geq 5$ & $\geq 10$ & $\geq 15$ & $\geq 20$ & $\geq 5$ & $\geq 10$ & $\geq 15$ & $\geq 20$ \\
\hline 83 & 56 & 32 & 19 & 85 & 97 & 99 & 100 \\
\hline 88 & 69 & 44 & 29 & 73 & 92 & 98 & 100 \\
\hline 100 & 89 & 61 & 42 & 70 & 90 & 97 & 100 \\
\hline 100 & 100 & 83 & 65 & 64 & 85 & 96 & 100 \\
\hline NPV & & & & PPV & & & \\
\hline \multicolumn{8}{|l|}{ ODI } \\
\hline$\geq 5$ & $\geq 10$ & $\geq 15$ & $\geq 20$ & $\geq 5$ & $\geq 10$ & $\geq 15$ & $\geq 20$ \\
\hline 87 & 75 & 66 & 63 & 80 & 94 & 96 & 100 \\
\hline 94 & 88 & 82 & 78 & 57 & 77 & 88 & 100 \\
\hline 100 & 97 & 91 & 88 & 44 & 68 & 85 & 100 \\
\hline 100 & 100 & 97 & 95 & 28 & 49 & 73 & 100 \\
\hline LR- & & & & LR+ & & & \\
\hline \multicolumn{8}{|l|}{ ODI } \\
\hline$\geq 5$ & $\geq 10$ & $\geq 15$ & $\geq 20$ & $\geq 5$ & $\geq 10$ & $\geq 15$ & $\geq 20$ \\
\hline 0,2 & 0,5 & 0,7 & 0,8 & 5,5 & 18,7 & 32,0 & $\infty$ \\
\hline 0,2 & 0,3 & 0,6 & 0,7 & 3,3 & 8,6 & 22,0 & $\infty$ \\
\hline 0,0 & 0,1 & 0,4 & 0,6 & 3,3 & 8,9 & 20,3 & $\infty$ \\
\hline 0,0 & 0,0 & 0,2 & 0,4 & 2,8 & 6,7 & 20,8 & $\infty$ \\
\hline
\end{tabular}




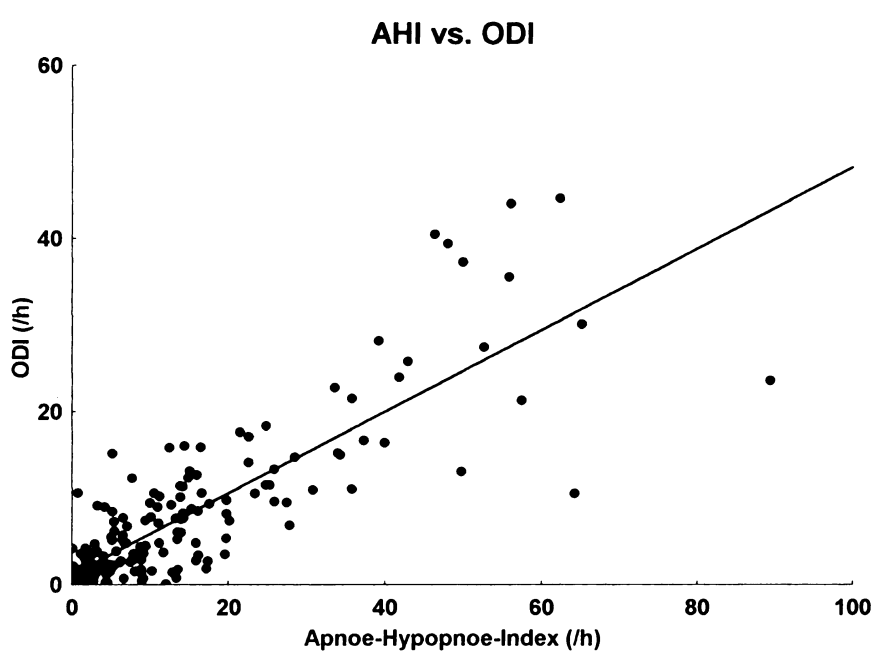

Abb. 2 Korrelation zwischen AHI und ODI.

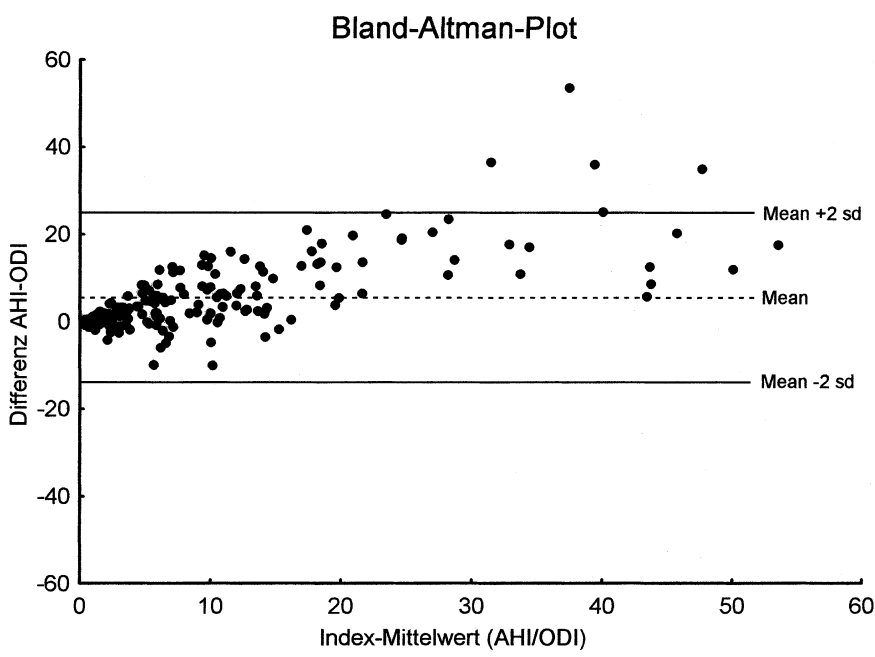

Abb. 3 Bland-Altman-Plot AHI vs. ODI.

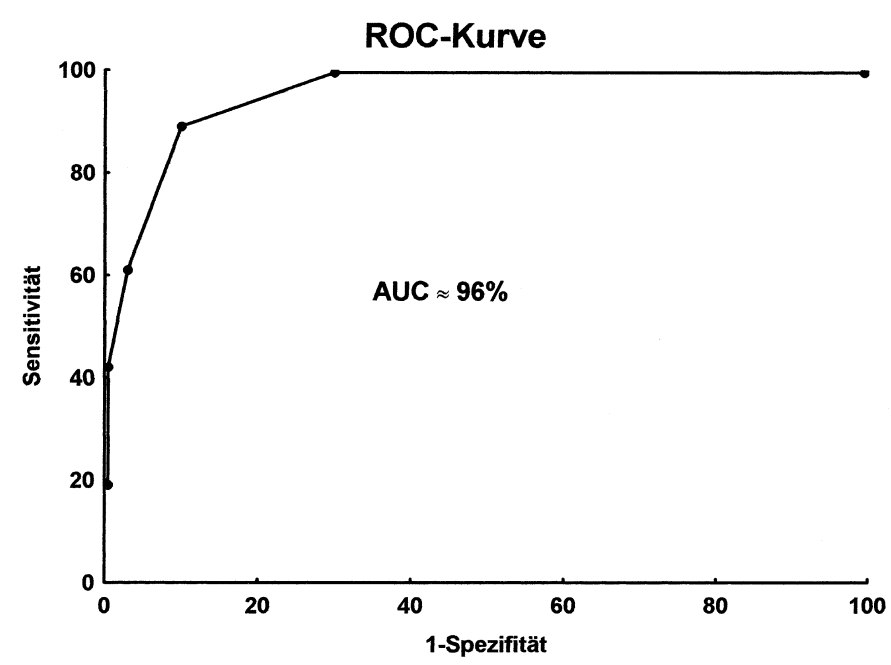

Abb. 4 ROC-Kurve. Definition einer SBAS als $A H I \geq 20$. 1-Spezifität gegen Sensivitität für verschiedene cut-off-Punkte des ODI. Die Fläche unter der Kurve (AUC) entspricht 96\%. der Differenzen beträgt 5,7 [95CI 4,4; 7,1]. Die Area under the Curve (AUC) der ROC-Kurve entspricht 96\% (Abb. 4).

\section{Diskussion}

Der Vergleich mit dem Goldstandard Polysomnographie lässt den Schluss zu, dass die automatische Erkennung kurzdauernder Desaturationen mit Abnahme der Sauerstoffsättigung von mindestens 4 Prozent im kontinuierlich während des Nachtschlafs aufgezeichneten Pulsoximetriesignal mit Hilfe der Software AHDip ein geeignetes Screening für schlafbezogene Atmungsstörungen bei Patienten mit Schlaganfall darstellt. Das Wahrscheinlichkeitsverhältnis (likelihood ratio) für ein positives Testergebnis bei einem Schwellenwert von 10 Entsättigungen pro Stunde liegt im Bereich von 7 bis 18 und ist demzufolge als ausreichend groß einzuschätzen [15]. Selbst bei Annahme einer niedrigen Prävalenz für SBAS von 20 bis 25 Prozent in einer Population von Patienten mit erstmaligem Schlaganfall würde ein positives Testergebnis die Posttestwahrscheinlichkeit in unserem Kollektiv immerhin auf $80 \%$ erhöhen.

Die Rolle der Pulsoximetrie als Screening für SBAS wird kontrovers diskutiert $[10,16]$. In Tab. 3 sind Studien zusammengestellt, in denen Polysomnographie und Pulsoximetrie simultan durchgeführt wurden [11,17-25]. Bei zeitlich getrennter Durchführung beider Untersuchungen resultiert eine schlechtere Korrelation mit dem Goldstandard, die hauptsächlich auf die erhebliche Nacht-zu-Nacht-Variabilität der leichten bis mittelschweren Schlafapnoe zurückzuführen ist $[24,26]$.

Die Population der Schlaganfallpatienten ist aus folgenden Gründen für ein pulsoximetrisches Screening auf SBAS geeignet: Da Schlaganfallpatienten in der Regel älter sind (in dieser Studie im Mittel 62 Jahre), ist die Sauerstoffsättigung aufgrund der altersgemäß oder darüber hinaus verminderten Lungenfunktion zu niedrigeren Werten und damit in den steilen Abschnitt der Sättigungscharakteristik verschoben [27]. Daraus resultiert, dass schon kürzere Atempausen zu einer messbaren Sauerstoffentsättigung führen, wodurch die Empfindlichkeit der Methode gesteigert wird.

Obwohl Schlaganfallpatienten mit SBAS seltener über Symptome einer obstruktiven Schlafapnoe klagen als jüngere Schlafapnoiker ohne Schlaganfall, bedingt die hohe Prävalenz der SBAS in dieser Population eine hohe Vortestwahrscheinlichkeit. Da der positive Vorhersagewert einer Untersuchungsmethode in erster Linie von der Spezifität und Prävalenz (= Vortestwahrscheinlichkeit) abhängt, dürfte die Aussagefähigkeit der Pulsoximetrie in der Akutphase nach Schlaganfall noch höher sein, da in der Akutphase nach einem Schlaganfall die Prävalenz einer SBAS noch höher ist $[2,4,28]$. Einschränkend muss jedoch gesagt werden, dass sich in der Frühphase nach Schlaganfall passager gehäuft zentrale Apnoen unter anderem vom Typ der CheyneStokes-Atmung finden, die nicht mit der für die obstruktive Schlafapnoe typischen ausgeprägt dreiecksförmigen Entsättigungscharakteristik, sondern einem sinusförmigen Verlauf der Sauerstoffsättigungswerte mit geringeren Schwankungen einhergehen [29]. Ob diese Entsättigungsphasen von unserer Soft- 
Tab. 3 Studien zur Validität der Pulsoximetrie als Screening für SBAS, in denen PSG und Pulsoximetrie simultan abgeleitet wurden

\begin{tabular}{|c|c|c|c|c|c|c|c|c|c|}
\hline Erstautor & $J a h r$ & $n$ & Cut-off ODI & $\begin{array}{l}\text { Desat ODI } \\
\text { (\%) }\end{array}$ & $\begin{array}{l}\text { Auswertung } \\
\text { Pulsoximetrie }\end{array}$ & Cut-off $A H I$ & $\begin{array}{l}\text { Desat AHI } \\
\text { (\%) }\end{array}$ & Sensitivität (\%) & Spezifität (\%) \\
\hline Farney ${ }^{7}$ & 1986 & 54 & $\mathrm{~N} / \mathrm{A}$ & 4 & manuell & & & 80 & 71 \\
\hline Douglas & 1992 & 200 & $5,10,15,20$ & 4 & automatisch & $\geq 15$ & & $67,53,41,36$ & $92,97,97,99$ \\
\hline Rauscher & 1993 & 116 & $\mathrm{~N} / \mathrm{A}$ & 3 & & $\geq 20$ & & 95 & 41 \\
\hline Duchna & 1995 & 207 & $\geq 5$ & $\mathrm{~N} / \mathrm{A}$ & & $\geq 5$ & 4 & 97 & 48 \\
\hline Levy $^{2}$ & 1996 & 301 & $\mathrm{~N} / \mathrm{A}$ & $\mathrm{N} / \mathrm{A}$ & & $\geq 15$ & $\mathrm{~N} / \mathrm{A}$ & 90 & 75 \\
\hline Lacassagne & 1997 & 329 & $\mathrm{~N} / \mathrm{A}$ & $\mathrm{N} / \mathrm{A}$ & & $\geq 15$ & & 90 & 58 \\
\hline Epstein & 1998 & 100 & $\geq 10$ & 4 & manuell & $>10$ & 4 & 74 & 90 \\
\hline Golpe & 1999 & 116 & 31,4 & 4 & automatisch & $\geq 10$ & 4 & 32 & 97 \\
\hline Olson ${ }^{2}$ & 1999 & 113 & $\mathrm{~N} / \mathrm{A}$ & $\mathrm{N} / \mathrm{A}$ & & $5,15,30$ & $\mathrm{~N} / \mathrm{A}$ & $88-92$ & $70-79$ \\
\hline Brouillette $^{3}$ & 2000 & 349 & & 4 & manuell & $\geq 1$ & 4 & 96 & 58 \\
\hline Vazquez & 2000 & 246 & $\geq 15$ & 4 & automatisch & $\geq 15$ & 4 & 98 & 88 \\
\hline Wessendorf & 2002 & 184 & $\geq 15$ & 4 & automatisch & $10,15,20,30$ & 4 & $32,44,61,83$ & $99,98,97,96$ \\
\hline
\end{tabular}

Anmerkungen:

$\mathrm{N} / \mathrm{A}$ : nicht angegeben oder nicht anwendbar

1 „apnea“ statt ODI

${ }^{2}$ Variabilitätsindex statt ODI

${ }^{3}$ ausschließlich pädiatrische Patienten

ware ebenfalls sensitiv erfasst werden, muss offen bleiben, da zentrale Apnoen in unserem Kollektiv eher selten nachgewiesen wurden.

Kriterien für ein Screening sind neben einer hohen Prävalenz die Chronizität der zu erkennenden Erkrankung, die Einfachheit und Genauigkeit der verwendeten Methode sowie der vorteilhafte Einfluss der Therapie auf Symptome und/oder Prognose. Zudem sollten die eingeleiteten Behandlungsmaßnahmen kostengünstig sein [30]. Die beiden ersten Voraussetzungen sind bei SBAS nach Schlaganfall erfüllt. Außerdem ist die Pulsoximetrie die einfachste und kostengünstigste derzeit verfügbare Methode zum Screening nach SBAS [10,23]. Die vorliegende Arbeit bestätigt, dass die Genauigkeit der Methode trotz automatischer Auswertung, welche den Aufwand der Methode ohne Zweifel weiter vereinfacht, für klinische Zwecke ausreichend ist. Aus den derzeit vorliegenden Studien geht hervor, dass die Diagnose einer nicht erkannten, koexistenten SBAS die Prognose des Schlaganfallpatienten verschlechtert $[5,6,31]$. Außerdem sprechen die Ergebnisse kleiner kontrollierter und unkontrollierter Serien dafür, dass die nasale CPAP-Therapie sich möglicherweise positiv auf die Symptomatik, die Lebensqualität und das kardiovaskuläre Risikoprofil auswirkt [7,32]. Offen ist dagegen, ob Morbidität und Mortalität dieser Patienten reduziert werden können.

Mögliche Nachteile der Methodik liegen zum einen in der fehlenden Information darüber, ob der Patient während der Aufzeichnung tatsächlich geschlafen hat, sowie in der Artefaktanfälligkeit der unkontrollierten Überwachung. Es ist davon auszugehen, dass eine bildschirmgestützte Analyse der Aufzeichnungsqualität, wie sie AHDip zulässt, die Validität sogar noch erhöhen könnte. Auf jeden Fall lässt sich anhand der am Bildschirm graphisch komprimiert dargestellten Signale aber rasch beantworten, ob die Pulsoximetrie wegen Artefakten wiederholt werden muss.
Die mit unserem Pulsoximeter und der Auswertesoftware AHDip erzielten Ergebnisse lassen sich nicht ohne weiteres auf andere Pulsoximeter übertragen. Darauf muss ausdrücklich hingewiesen werden, da technisch unterschiedlich konzipierte Pulsoximeter zur Standardausstattung einer Intensivstation, stroke unit oder internistischen Abteilung gehören, sich aber nicht unbedingt für das Screening nach SBAS eignen. Voraussetzung für die hohe Validität des Pulsoximeters als Screeningverfahren ist nämlich nicht nur die artefaktfreie Aufzeichnung der Signale, sondern in erster Linie die Güte der Signalverarbeitung. Zu fordern ist eine hohe Dichte der gespeicherten Messwerte (zum Beispiel $1 \mathrm{~Hz}$ ) und eine allenfalls minimale Filterung der Rohsignale [33]. In diesem Zusammenhang sei darauf hingewiesen, dass gerade Pulsoximeter in Monitoringsystemen auf Intensivstationen nicht selten zur Vermeidung von Fehlalarmen geräteseitig gedämpft und demzufolge für das Screening auf SBAS ungeeignet sind.

In Zukunft sollte geprüft werden, ob die zusätzliche Analyse der Herzfrequenzvariabilität, die als Zeichen der sympathischen Aktivierung infolge der Apnoen bekanntermaßen erhöht ist, die Validität des hier beschriebenen Verfahrens weiter verbessern kann [34]. Keinesfalls ist aber die propagierte Ermittlung des Signalvariabilitätsindex oder der Zeit mit Sättigungswerten unter $90 \%$ der von uns entwickelten Methode überlegen. Dies gilt insbesondere für Patienten mit zahlreichen kurzen Apnoen oder zentralen Apnoen, bei denen sich oft keine ausgeprägten Entsättigungen unter $90 \%$ finden $[21,24]$.

Zusammenfassend konnte in dieser Studie gezeigt werden, dass die Pulsoximetrie mit hoher Datendichte und weitgehend ungefiltertem Signal in Verbindung mit der automatischen Auswertesoftware AHDip ein gut geeignetes Screening für SBAS nach Schlaganfall darstellt, wenn es um die Erkennung mittelschwerer bis schwerer Apnoeformen geht. 


\section{Literatur}

${ }^{1}$ Mohsenin V, Valor R. Sleep apnea in patients with hemispheric stroke. Arch Phys Med Rehabil 1995; 76: 71 - 76

2 Bassetti C, Aldrich MS. Sleep apnea in acute cerebrovascular diseases: final report on 128 patients. Sleep 1999; 22: 217-223

${ }^{3}$ Wessendorf TE, Teschler $\mathrm{H}$ et al. Sleep-Disordered Breathing Among Patients With First-Ever Stroke. J Neurol 2000; 247: 41 - 47

${ }^{4}$ Parra O, Arboix A et al. Time Course of Sleep-related Breathing Disorders in First-Ever Stroke or Transient Ischemic Attack. Am J Resp Crit Care Med 2000; 161: 375-380

${ }^{5}$ Dyken ME, Somers VK et al. Investigating the relationship between stroke and obstructive sleep apnea. Stroke 1996; 27: 401 - 407

${ }^{6}$ Good DC, Henkle JQ et al. Sleep-disordered breathing and poor functional outcome after stroke. Stroke 1996; 27: 252 - 259

7 Wessendorf TE, Wang YM et al. Treatment of obstructive sleep apnoea with nasal continuous positive airway pressure in stroke. Eur Respir J 2001; 18: 623-629

${ }^{8}$ Sandberg O, Franklin KA et al. Sleep apnea, delirium, depressed mood, cognition, and ADL ability after stroke. J Am Geriatr Soc 2001; 49: $391-397$

${ }^{9}$ Mohsenin V. Sleep-related breathing disorders and risk of stroke. Stroke 2001; 32: $1271-1278$

${ }^{10}$ Netzer N, Eliasson AH et al. Overnight pulse oximetry for sleep-disordered breathing in adults: a review. Chest 2001; 120: 625-633

${ }^{11}$ Vazquez JC, Tsai WH et al. Automated analysis of digital oximetry in the diagnosis of obstructive sleep apnoea. Thorax 2000; 55: $302-307$

12 Rechtschaffen A, Kales A. A manual of standardized terminology: Techniques and scoring system for sleep stages of human subjects. 1968

${ }^{13}$ American Sleep Disorders Association. EEG arousals: scoring rules and examples. Sleep 1992; 15: 174-184

${ }^{14}$ Bland JM, Altman DG. Statistical methods for assessing agreement between two methods of clinical measurement. Lancet 1986; 1 : $307-310$

15 Jaeschke R, Guyatt GH, Sackett DL. Users' guides to the medical literature. III. How to use an article about a diagnostic test. B. What are the results and will they help me in caring for my patients? The EvidenceBased Medicine Working Group. JAMA 1994; 271: 703-707

${ }^{16}$ Practice parameters for the use of portable recording in the assessment of obstructive sleep apnea. Standards of Practice Committee of the American Sleep Disorders Association [see comments]. Sleep 1994; 17: $372-377$

${ }^{17}$ Farney RJ, Walker LE et al. Ear oximetry to detect apnea and differentiate rapid eye movement (REM) and non-REM (NREM) sleep. Screening for the sleep apnea syndrome. Chest 1986; 89: 533-539

${ }^{18}$ Douglas NJ, Thomas JM. Clinical value of polysomnography. Lancet 1992; 339: $347-385$
${ }^{19}$ Rauscher H, Popp W, Zwick H. Model for investigating snorers with suspected sleep apnea. Thorax 1993; 48: 275-279

${ }^{20}$ Duchna HW, Rasche K et al. Sensitivität und Spezifität der Pulsoximetrie in der Diagnostik schlafbezogener Atmungsstörungen. Pneumologie 1995; 49 Suppl 1: 113-115

${ }^{21}$ Levy P, Pepin JL et al. Management of simple snoring, upper airway resistance syndrome, and moderate sleep apnea syndrome. Sleep 1996; 19: S101 - S110

${ }^{22}$ Lacassagne L, Didier A et al. Role of nocturnal oximetry in screening for sleep apnea syndrome in pulmonary medicine. Study of 329 patients. Rev Mal Respir 1997; 14: 201 - 207

${ }^{23}$ Epstein LJ, Dorlac GR. Cost-effectiveness analysis of nocturnal oximetry as a method of screening for sleep apnea-hypopnea syndrome. Chest 1998; 113: 97-103

24 Olson LG, Ambrogetti A, Gyulay SG. Prediction of sleep-disordered breathing by unattended overnight oximetry. J Sleep Res 1999; 8: $51-55$

25 Brouillette RT, Morielli A et al. Nocturnal pulse oximetry as an abbreviated testing modality for pediatric obstructive sleep apnea. Pediatrics 2000; 105: 405-412

${ }^{26}$ Hein $\mathrm{H}$, Behnke $\mathrm{G}$ et al. The therapeutic effect of theophylline in mild obstructive sleep Apnea/Hypopnea syndrome: results of repeated measurements with portable recording devices at home. Eur J Med Res 2000; 5: 391 - 399

${ }^{27}$ Gries RE, Brooks LJ. Normal oxyhemoglobin saturation during sleep. How low does it go? Chest 1996; 110: 1489-1492

28 Iranzo A, Santamaria J et al. Prevalence and clinical importance of sleep apnea in the first night after cerebral infarction. Neurology 2002; 58: 911 - 916

${ }^{29}$ Ullmer E, Strobel WM, Soler M. Cheyne-stokes respiration or obstructive sleep apnoea: patterns of desaturation [letter]. Respiration 2000; 67: 203

${ }^{30}$ Baumel MJ, Maislin G, Pack AI. Population and occupational screening for obstructive sleep apnea: Are we there yet? Am J Resp Crit Care Med 1997; 155: 9-14

${ }^{31}$ Spriggs DA, French JM et al. Snoring increases the risk of stroke and adversely affects prognosis. QJM 1992; 83: 555-562

32 Sandberg O, Franklin KA et al. Nasal continuous positive airway pressure in stroke patients with sleep apnoea: a randomized treatment study. Eur Respir J 2001; 18: 630-634

${ }^{33}$ Wiltshire N, Kendrick AH, Catterall JR. Home oximetry studies for diagnosis of sleep apnea/hypopnea syndrome: limitation of memory storage capabilities. Chest 2001; 120: 384-389

34 Zamarron C, Romero PV et al. Screening of obstructive sleep apnoea: heart rate spectral analysis of nocturnal pulse oximetric recording. Respir Med 2001; 95: 759-765 